\title{
Capsid coding sequences of foot-and-mouth disease viruses are determinants of pathogenicity in pigs
}

\author{
Louise Lohse $^{1^{*}}$, Terry Jackson ${ }^{2}$, Anette Bøtner ${ }^{1}$ and Graham J Belsham ${ }^{1}$
}

\begin{abstract}
The surface exposed capsid proteins, VP1, VP2 and VP3, of foot-and-mouth disease virus (FMDV) determine its antigenicity and the ability of the virus to interact with host-cell receptors. Hence, modification of these structural proteins may alter the properties of the virus.

In the present study we compared the pathogenicity of different FMDVs in young pigs. In total 32 pigs,

7-weeks-old, were exposed to virus, either by direct inoculation or through contact with inoculated pigs, using cell culture adapted (O1K B64), chimeric (O1K/A-TUR and O1K/O-UKG) or field strain (O-UKG/34/2001) viruses. The O1K B64 virus and the two chimeric viruses are identical to each other except for the capsid coding region. Animals exposed to O1K B64 did not exhibit signs of disease, while pigs exposed to each of the other viruses showed typical clinical signs of foot-and-mouth disease (FMD). All pigs infected with the O1K/O-UKG chimera or the field strain (O-UKG/34/2001) developed fulminant disease. Furthermore, 3 of 4 in-contact pigs exposed to the O1K/O-UKG virus died in the acute phase of infection, likely from myocardial infection. However, in the group exposed to the O1KA-TUR chimeric virus, only 1 pig showed symptoms of disease within the time frame of the experiment (10 days). All pigs that developed clinical disease showed a high level of viral RNA in serum and infected pigs that survived the acute phase of infection developed a serotype specific antibody response. It is concluded that the capsid coding sequences are determinants of FMDV pathogenicity in pigs.
\end{abstract}

\section{Introduction}

Foot-and-mouth disease (FMD) is one of the world's most economically important infectious diseases of farm animals including cattle, pigs and sheep. The aetiological agent of FMD is foot-and-mouth disease virus (FMDV) which is the prototype Aphthovirus within the family Picornaviridae. The virus can infect about 70 different wild life species and can spread rapidly causing high morbidity but only low mortality except in young animals [1]. The severity of the disease varies between hosts with pigs and cattle exhibiting clear clinical signs while infection in sheep is often difficult to detect by clinical observation. The disease in cattle and pigs follows a rapid time course and causes a rise in body temperature and the development of vesicular lesions in and around

\footnotetext{
* Correspondence: loloh@vet.dtu.dk

${ }^{1}$ National Veterinary Institute, Technical University of Denmark, Lindholm, Kalvehave DK-4771, Denmark

Full list of author information is available at the end of the article
}

the mouth and on the feet. Infected animals display varying degrees of salivation, inappetence and lameness according to the severity of lesions. The clinical course of the infection usually subsides within 7-14 days and is accompanied by a rapid generation of neutralizing antibodies within the serum. However, many animals can subsequently "carry" the virus in the oropharynx for a prolonged period; this "carrier state" can be maintained for several months (in sheep) or years (in cattle and buffalo). In general it is considered that pigs do not become carriers [1] although there is some evidence to the contrary [2-4].

FMDV exists in 7 distinct serotypes, O, A, C, SAT1, SAT2, SAT3 and Asia-1. Each virus particle contains a positive sense single-stranded RNA genome of about $8.4 \mathrm{~kb}$ enclosed within a protein shell consisting of 60 copies each of the 4 capsid proteins 1A (VP4), 1B (VP2), 1C (VP3) and 1D (VP1) [5]. VP1, VP2 and VP3 are exposed on the outer surface of the virus particle and

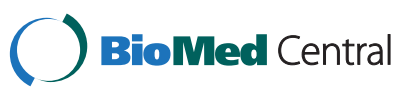


hence they determine both the antigenicity of the virus and its ability to interact with specific receptors on cells.

The major cellular receptor for FMDV is believed to be the integrin $\alpha v \beta 6$ which is expressed on epithelial cells [6-8] but other RGD-binding integrins, $\alpha v \beta 8, \alpha v \beta 1$ and $\alpha v \beta 3$ ), have also been shown to function as receptors for FMDV in cell culture [8-11]. Cell culture adapted serotype $\mathrm{O}$ viruses can also bind to cells via heparan sulfate (HS) and can use this interaction to initiate infection. The ability to bind to HS is associated with specific amino acid substitutions (including His56 to Arg56 in VP3) on the surface of the virus [12-14]. However, the HS binding phenotype has been linked to attenuation of serotype $O$ virus in cattle $[14,15]$.

Different strains of FMDV can vary greatly in their pathogenicity within different host species. For instance, some virus strains (e.g. O/Taiwan 1997) cause disease in pigs but not in cattle (i.e. the porcinophilic strains) [16]. Some of these viruses (namely O/Taiwan 1997) have been shown to contain a deletion within the coding region for the $3 \mathrm{~A}$ protein $[17,18]$; however, other porcinophilic strains from Korea (O/SKR/AS/2002) had an intact 3A coding region [19].

Full-length infectious cDNAs corresponding to the FMDV genome have been produced for serotype $\mathrm{O}$ [20$22]$ and serotype A $[23,24]$ viruses. These have permitted the construction of chimeric full-length infectious FMDV cDNAs in which the capsid coding sequences of one virus has been replaced with the equivalent coding region of a different virus. Viable chimeric viruses have been rescued from these cDNAs and been shown to display the antigenicity of the introduced capsid sequences $[14,15,25,26]$.

Previously we have replaced the coding region for the surface exposed capsid proteins (VP1, VP2 and VP3) of an infectious cDNA derived from a cell culture adapted, HS-binding, strain of O1 Kaufbeuren (O1K B64) (pT7S3, see [22]) with the corresponding region of field strains of serotype $\mathrm{O}(\mathrm{O} / \mathrm{UKG} / 34 / 2001)$ or serotype A (A-Tur/06) viruses [15]. Thus the chimeric viruses obtained (O1K/O-UKG and O1K/A-Tur) and the parental $\mathrm{O} 1 \mathrm{~K}$ B64 are identical to each other except for the capsid coding region. In the previous study, these chimeric viruses were shown to be pathogenic in cattle whereas the parental O1K B64 strain is completely attenuated in this host [15]. Thus the production of chimeric viruses can be a useful tool for identifying determinants of pathogenicity.

Since the pathogenicity of FMDVs can vary between hosts we have now determined the virulence of these chimeric viruses and their parent $(\mathrm{O} 1 \mathrm{~K}$ B64) within pigs as well. For comparison the O/UKG/34/2001 field virus was tested in parallel. This virus produces a relatively mild form of the disease in cattle [27] but has also been well characterized within experimental studies in pigs in which it causes quite severe disease [28,29].

Here we report that the rescued cell culture adapted O1K B64 virus is also highly attenuated in pigs. However, the two chimeric virus derivatives, O1K/O-UKG and O1K/A-TUR, were able to cause typical clinical signs of FMD in inoculated pigs albeit with different efficiencies. Some young pigs infected by contact exposure to the O1K/O-UKG chimera died suddenly during the acute phase of infection. These studies show that the capsid coding sequences are determinants of FMDV pathogenicity in pigs.

\section{Material and methods}

\section{Animal experiments and samples}

Experimental procedures and animal management protocols were carried out in accordance with the requirements of the Danish Animal Experimentation Inspectorate, license no. 2008/561-1541. All pigs were housed in the high containment BSL3+ experimental facilities at the National Veterinary Institute, Lindholm.

In total, 32 weaner pigs ( 7 weeks of age) were obtained from a conventional Danish swine herd with specific pathogen free (SPF) status, including freedom from enzootic pneumoniae, atrophic rhinitis, swine dysentery, porcine reproductive and respiratory syndrome and most serotypes of pleuropneumoniae. All pigs were found to be healthy by veterinary inspection on arrival at the Institute, 1 week before the start of the experiment. All pigs were fed a commercial diet for weaning pigs and water was provided ad libitum. Straw was used for bedding.

Pigs were randomly divided into 4 groups of 8 pigs and the individual groups were kept in separate pens with no physical contact between them. Groups 1 and 2, in one stable, shared the same air space while Groups 3 and 4, in another stable, also shared air space with each other. Four pigs in each group were inoculated with the different FMDVs: Group 1 was inoculated with the cell culture adapted B64 strain of the O1 Kaufbeuren virus (O1K B64); Group 2, was inoculated with the O1K/ATUR chimeric virus [15], a derivative of O1K B64 containing the surface exposed capsid proteins (VP1, VP2 and VP3) from A/Turkey 2/2006; Group 3 was inoculated with a second chimeric virus (O1K/O-UKG) [15] also derived from $\mathrm{O} 1 \mathrm{~K}$ B64 and containing VP1, VP2 and VP3 from O UKG/34/2001 and Group 4 was inoculated with the field strain O UKG/34/2001, as used previously at Lindholm, in cattle by Stenfeldt et al. [27]. Inoculation was performed in the heel bulb, each pig receiving approximately $10^{5} \mathrm{TCID}_{50}$ in a volume of $0.3 \mathrm{~mL}$. All inoculated pigs were anaesthetized during the process. The remaining four pigs in each group were left as in-contact animals. 
On each subsequent day, all pigs were examined for clinical signs of FMD, to generate a clinical score, and rectal body temperatures were recorded. Clinical scores (CS) were assigned based on a semi-quantitative scoring system with each animal being assessed on 4 FMDrelevant parameters (general well-being, appetite, lameness and the presence of lesions on the mouth/nose/ tongue and feet). Body weight was recorded on arrival at the Institute and at the termination of the experiment. Blood samples were collected from the jugular vein on the following days post infection (dpi) $0,1,2,3,4,5,7$ and 10 for inoculated pigs and on dpi $0,4,5,6,7,8,9$ and 10 for contact pigs. Oral swab samples were collected at the same times. Pigs, which developed significant lesions, were treated with analgesics. Pigs that displayed clinical symptoms which reached the humane endpoint criteria during the course of the experiment, were euthanised when necessary and, after 10 days, surviving pigs were euthanised by intravenous injection of sodium pentobarbital. All pigs were autopsied and relevant tissues were collected for further examination.

\section{Quantification of anti-FMDV antibodies using a solid phase blocking ELISA}

Serum samples were screened for antibodies to FMDV in a solid phase blocking ELISA [30,31]. In brief, microtitre plates were coated with guinea-pig immune serum raised against FMDV serotype $O$ or serotype A, as appropriate, before addition of the respective inactivated FMDV antigen. Test serum samples were then added and incubated overnight, the plates were washed and then rabbit anti-FMDV serotype $\mathrm{O}$ or $\mathrm{A}$ serum was added. Bound rabbit antibodies were detected with horseradish-peroxidase conjugated pig anti-rabbit IgG (Dakopatts P0217, DakoCytomation, Denmark A/S). All serum samples were initially screened at a dilution of 1:5 and positive samples were further analyzed in a two-fold titration, starting at a dilution of 1:10 to allow determination of antibody titers.

\section{Quantification of viral RNA using a quantitative real time RT-PCR}

FMDV RNA in serum, oral swabs and heart tissue was determined using a quantitative real-time RT-PCR assay [32] which is targeted to the 5'-untranslated region of the viral RNA. In brief, total RNA was extracted from $200 \mu \mathrm{L}$ of sample using a MagNa Pure LC Total Nucleic Acid Isolation Kit (Roche) according to the manufacturer's instructions in an automated RNA extraction system. After extraction, each sample was eluted in 50 $\mu \mathrm{L}$ Elution Buffer. cDNA was made using $6 \mu \mathrm{L}$ of extracted RNA in a total volume of $15 \mu \mathrm{L}$, using a TaqMan RT kit mastermix with random hexamer primers (Applied Biosystems) at $48^{\circ} \mathrm{C}$ for $45 \mathrm{~min}$ and $95^{\circ} \mathrm{C}$ for
5 min. Seven $\mu \mathrm{L}$ of cDNA was mixed with $18 \mu \mathrm{L}$ of $2 \times$ TaqMan universal PCR mastermix (Applied Biosystems) containing $22.5 \mathrm{pmol}$ of each primer and $7.5 \mathrm{pmol}$ of fluorescently labeled probe. PCR amplification was carried out for 50 cycles in a Thermal cycler (Mx3005P, Stratagene) and analysed using MxPro qPCR software (Stratagene). Standard curves were produced using 10fold dilutions $\left(10^{0}-10^{7}\right)$ of the synthesis control (an RNA transcript from a plasmid containing part of the $5^{\prime}$ untranslated region of FMDV strain O/UKG/34/2001).

\section{Sequencing of viral RNA}

To determine if cross contamination between groups sharing the same air space had occurred, sequencing of viral RNA isolated from serum and heart tissue from pigs infected with the O1K/O-UKG (Group 3) and OUKG/34/2001 (Group 4) viruses was performed. In brief, random primed cDNA was made, as described previously [33] and PCR products corresponding to most of the VP1 (ca. $520 \mathrm{bp}$ ) and to part of the VP4/VP2 coding regions (ca. $820 \mathrm{bp}$ ) were amplified and sequenced in both directions. Sequences generated were compared to those known for O1Kaufbeuren [GenBank: X00871] and O/UKG/35/2001 [GenBank: AJ539141] using BLASTN.

\section{Statistics}

Data analysis was performed using GraphPad In Stat version 3.00 (GraphPad Software, San Diego, CA, USA). One way ANOVA with post test was selected for comparison of mean body weights between groups.

\section{Results}

\section{Clinical findings}

Four pigs within each of four groups of pigs were inoculated with different FMDVs and, within each group, four other pigs were left as "in-contact" pigs (see Materials and methods). Clinical signs of FMD within the pigs varied between the groups exposed to the cell culture adapted O1KB64, chimeric (O1K/O-UKG and O1K/ATUR) or field strain (O-UKG/34/2001) viruses. Clinical scores for the individual pigs within the 4 groups are shown in Figure 1. The results show that pigs inoculated with the cell culture adapted virus, O1K B64, or exposed to it by direct contact with the inoculated pigs (Group 1 ), did not develop any clinical signs of FMD. In contrast, pigs in each of the 3 other groups developed moderate or severe symptoms of FMD, including vesicular lesions in the oral cavity and also on the feet (mainly along the coronary band and in the heel bulb area) which resulted in lameness. Pigs affected by disease typically showed slight depression and loss of appetite, while only sporadic temperature increases in individual pigs were observed (data not shown). 


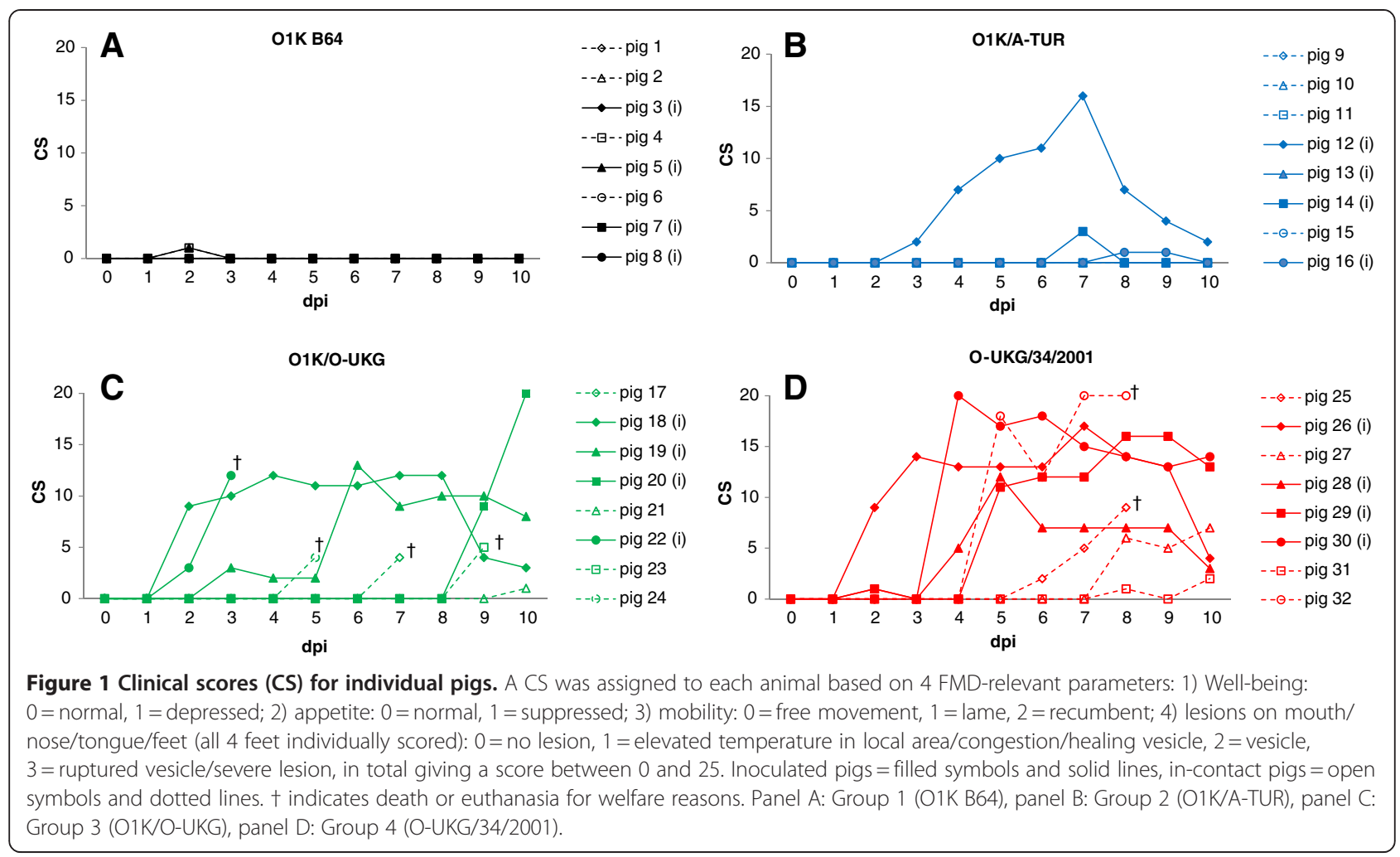

In more detail, only one pig in Group 2 (pig 12) which was inoculated with the chimeric serotype A virus (O1K/A-TUR) developed severe disease with a maximum clinical score of 16 (see Figure 1b). Clinical disease was not observed in the contact pigs in the 10 day experiment (but see below). However, all pigs (in Group 3) exposed to the O1K/O-UKG chimeric virus (inoculated and contacts) developed FMD, including vesicles in the oral cavity and on the feet, with clinical scores of up to 20 (Figure 1c). In this Group, one inoculated pig (id no. 22), and 3 of the 4 contact pigs (id nos. 17, 23, 24) were found dead in the pen only one day after the first appearance of clinical signs. The remaining contact pig (id no. 21) did not develop vesicular lesions indicative of FMD, however, at dpi 10 (the day of termination of the experiment) lameness was recorded. Pigs in group 4 exposed by inoculation or through contact to the $\mathrm{O}$ UKG/34/2001 field strain all developed apparent clinical disease with clinical scores of between 12 and 20 (Figure 1d). Two contact pigs in this group (id nos. 25 and 32) were euthanised on dpi 8 for animal welfare reasons, as administration of analgesics did not appear to relieve their pain.

Growth rate, defined as the weight difference between the start and termination of the experiment was calculated for the individual pigs. Mean weight difference for the 4 groups were compared and no significant difference between groups were found ( $p>0.05$; data not shown).
Weight gain for pigs included in the experiment for the full time period was on average $2.3 \mathrm{~kg}$. Pigs, which died or were euthanised before the conclusion of the experiment, were not weighed, however, by visual inspection at the post mortem (PM) examination these pigs appeared to be of fair condition.

\section{Autopsy}

PM examination revealed mostly lesions or healed lesions on the feet and in the oral cavity typical of FMD. In one pig (id no. 11), however, light grey areas on the left ventricle, compatible with myocardial degeneration in the heart, was observed. This finding was not related to any signs of clinical illness, but this pig showed rising levels of viral RNA in serum (see below) on the last days before termination of the experiment and could therefore have been at risk of acute death from myocardial infarction, if the experiment had been prolonged.

\section{Virus detection in serum}

The level of FMDV RNA was determined in serum samples collected at different time points throughout the course of the experiment (Figure 2). Inoculated pigs, which showed clinical signs of disease, had high levels of FMDV RNA (up to $10^{7}$ genome copies $/ \mu \mathrm{L}$ ) from dpi 1 , reflecting rapid replication and systemic dissemination of the virus. For all pigs with clinical symptoms of FMD (inoculated and contacts) viral RNA was detected in 


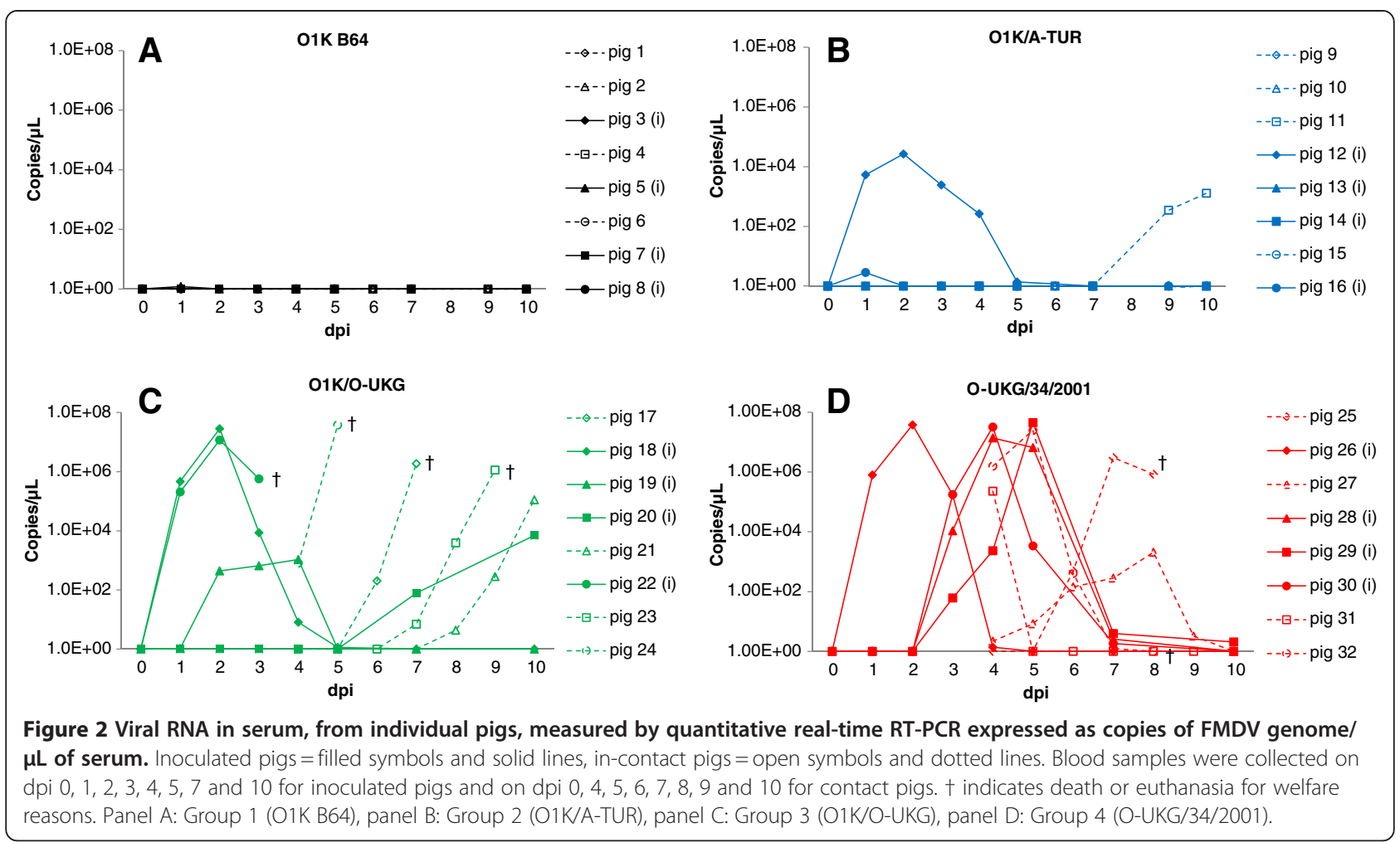

serum for a period of 4-6 days. Typically, viral RNA was detected in serum for one day before clinical signs appeared. The results from each Group will be considered below in more detail.

\section{K B64 (Group 1)}

No clinical signs of FMD were observed in this group but one out of 4 pigs (id no. 5) inoculated with the cell culture adapted virus had detectable but very low amounts of viral RNA (1.2 genome copies/ $\mu \mathrm{L}$ ) in serum for one day only (Figure 2a). All other pigs in this Group (inoculated and contacts) had no detectable viral RNA in their serum. Thus there was very limited evidence for any replication and no sign of any transmission of the virus from inoculated to contact pigs.

\section{O1K/A-TUR (Group 2)}

In the pigs inoculated with the O1K/A-TUR chimeric virus, one inoculated pig (id no. 12), which showed clinical disease, had relatively high levels of viral RNA in serum from dpi 1-5 (up to $10^{4}$ genome copies $/ \mu \mathrm{L}$ ). One other inoculated pig (id no. 16) had a very low level of viral RNA in serum (2.84 genome copies/ $\mu \mathrm{L}$ ) for one day after inoculation, however, no clear clinical symptoms were ever observed in this pig. In addition, one contact pig (id no. 11) had rising levels (up to $10^{3}$ genome copies $/ \mu \mathrm{L}$ ) of FMDV RNA, starting at dpi 9 (Figure 2b). However, as the experiment was terminated on dpi 10, the development of the infection in this pig could not be followed but, as indicated above, this pig did exhibit signs of myocardial degeneration in the heart at autopsy.

\section{O1K/O-UKG (Group 3)}

Pigs inoculated with the O1K/O-UKG chimeric virus, all showed clinical signs of FMD and had viral RNA detectable in serum for up to five days. Three out of 4 inoculated pigs (id nos. 18, 19, 22) were positive from dpi 1, while the fourth (id no. 20) became positive during the same time period as the contacts, i.e. from dpi 4 and onwards. The sudden deaths of one of the inoculated pigs and three of the four in-contact pigs clearly precluded detailed analysis of the progression of the infection in these animals, however, all these 4 pigs had high levels of FMDV RNA $\left(10^{5}\right.$ to $10^{7}$ genome copies $\left./ \mu \mathrm{L}\right)$ in their serum on the day before they died (Figure 2c).

\section{O-UKG/34/2001 (Group 4)}

In the Group infected with the field strain O-UKG/34/ 2001, one inoculated pig (id no. 26) was viral RNA positive in serum on dpi 1, while the remaining 3 inoculated pigs (id nos. 28, 29, 30) were positive from dpi 3 (all up to $10^{7}$ genome copies $/ \mu \mathrm{L}$ ), see Figure $2 \mathrm{~d}$. Contact pigs in this group were identified as positive for virus RNA from dpi 4 and onwards, but could have been positive at an earlier time point since the first serum samples from the contact 
pigs were only taken at dpi 4 after the null-sample (for animal welfare reasons). Therefore we do not know the true time point for the first appearance of virus in the blood of contact pigs tested positive on this day.

Whether a delayed infection or transmission of virus by contact was the case for some of the inoculated pigs (id nos. 28, 29, 30 in Group 4 and also id no. 20 in Group 3) that responded "late" (i.e. 3-7 days after inoculation) in this experiment is not clear.

\section{Detection of FMDV RNA in oral swab samples}

The level of FMDV RNA in oral swab samples showed a pattern consistent with the detection of viral RNA in serum, but at lower levels (c.f. Figure 2 and Figure 3). The Ct-values indicated about a 100-fold lower virus load in oral swab samples compared to serum and we believe that a more precise quantification of the virus in swab samples from the mouth is not possible (e.g. due to variable level of salivation etc.). However, detectable viral RNA in oral swab samples was typically observed over a longer time span than in serum, often 6-9 days. In fact, for the pigs containing the highest levels of virus (pigs in Groups 3 and 4) the oral swab samples still contained detectable levels of FMDV RNA at the day of termination (dpi 10). Furthermore, FMDV RNA was found at low levels sporadically in the oral swab samples of several pigs not going through a systemic FMDV infection in the groups inoculated with virus of low virulence, i.e. O1K B64 and O1K/A-TUR infected groups (Figure 3).

\section{FMDV RNA in heart tissue}

Due to the sudden death of several pigs from Group 3 (exposed to the O1K/O-UKG chimera), the presence of FMDV RNA was assayed in heart tissue from pigs infected with this virus and (for comparison) in samples from pigs in Group 4 (exposed to the O-UKG/34/2001 field strain). Based on Ct-values, the pigs could be divided into 3 sets. The first set was comprised of the pigs that died in the acute state of infection, after showing initial clinical symptoms (id nos. 17, 22, 23, 24). These pigs had high levels of FMDV RNA (Ct-values $=17-18$ ) in heart tissue as well as in serum (Ct-values $=14-19)$. The two other sets included pigs that survived until the end of the experiment. The first of these sets included pigs that developed infection late in the experimental period, i.e. first detectable level of viral RNA in serum at dpi 7-9 (id nos. 20, 21, 25). These pigs were positive for detection of FMDV RNA in heart tissue $(\mathrm{Ct}=23-33)$ and serum $(\mathrm{Ct}=17-26)$, but had a lower load of virus than pigs that died in the acute phase of infection. The second set of pigs that survived until the

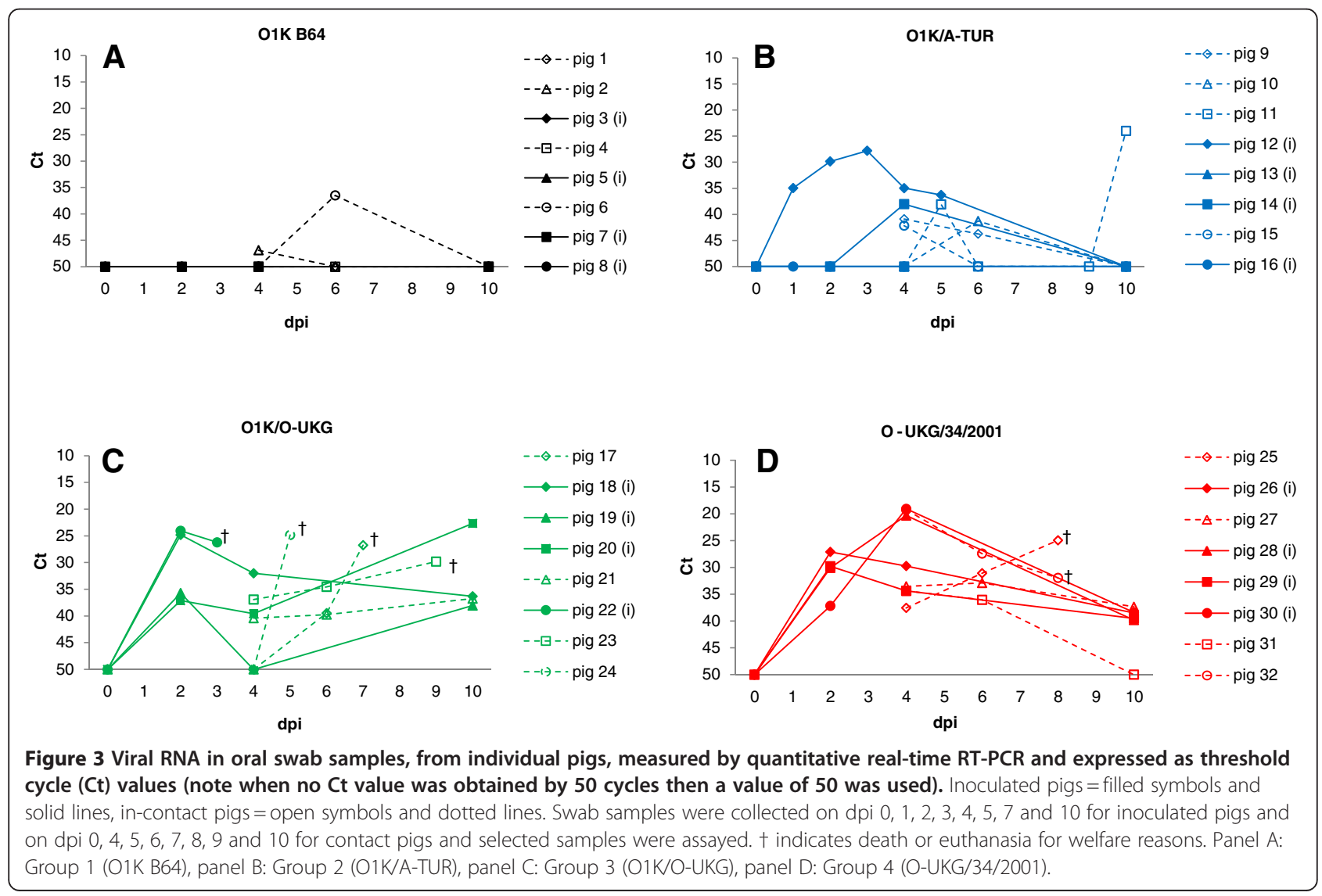


end of the experiment included pigs that were past or almost past the acute phase of infection (id nos. 18, 19, 26, $27,28,29,30,31,32$ ). These pigs were negative or had low levels of virus RNA by RT-PCR analysis for FMDV RNA in both heart tissue and serum at this stage.

\section{Sequencing of viral RNA isolated from serum and heart tissue}

Pigs within Groups 3 (O1K/O-UKG chimera) and 4 (OUKG/34/2001 field strain) were housed within the same air space although they were well separated (by over $3 \mathrm{~m}$ ); previous studies have shown that a physical gap of $70 \mathrm{~cm}$ [34] is sufficient to prevent spread of virus by aerosol between pigs. However, we wanted to ensure that the clinical results observed reflected the response to the specific virus used for that Group. Thus, RNA was extracted from serum and heart tissue samples and used to amplify, by RT-PCR, fragments corresponding to most of the VP1 coding region (ca. $520 \mathrm{bp}$ ), that is common to each of these viruses, and to part of the VP4/ VP2 coding region (ca. $820 \mathrm{bp}$ ), which includes the junction between the O1K B64 and the O-UKG capsid coding sequence in the chimera. As expected, the VP1 sequences were identical to the O-UKG/35/2001 sequence [GenBank: AJ539141] in each of the 3 samples tested (from pigs with id nos. 23, 24 and 25). However, around the junction between the VP4 and VP2 coding sequences, RNA obtained from pigs with id nos. 23 and 24 (Group 3) matched the expected structure of the O1K/O-UKG chimera while the sequence generated from the pig with id no. 25 sample (Group 4) exactly matched the O-UKG/35/2001 sequence (data not shown). Thus these results indicated that these pigs had indeed been infected with the expected virus.

\section{Development of FMDV specific antibodies}

Anti-FMDV specific antibodies were produced in pigs which developed a systemic FMD infection and survived. Three to five days after virus was detected in serum, antibodies with the expected serotype specificity $(\mathrm{O}$ or A) could be demonstrated. The diagnostic cut-off level, defined as $45 \%$ (for serotype A) or 50\% (for serotype O) blocking of the serotype-specific antigen in ELISA, was reached after 5-7 days (Figure 4). Contact pigs which died acutely in the group of O1K/O-UKG infected pigs did not survive long enough to develop such antibodies. No antibodies were detected in animals that did not exhibit signs of clinical disease.

\section{Discussion}

The pathogenicity in young pigs of 4 different, genetically defined, FMDVs has been compared. The properties of the cell culture adapted $\mathrm{O} 1 \mathrm{~K}$ B64 virus and its chimeric derivatives (O1K/O-UKG and O1K/A-TUR)

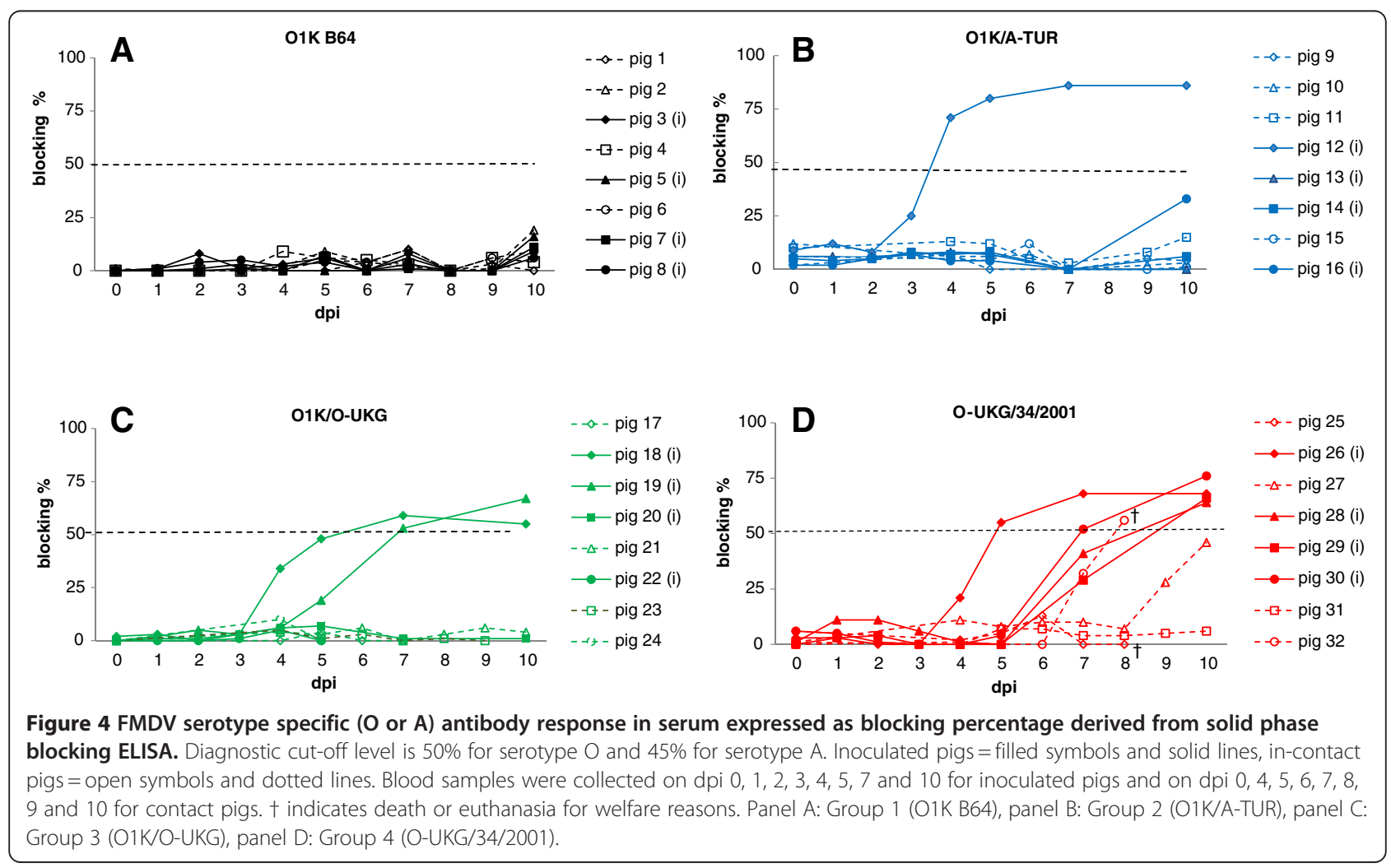


have been investigated in cattle previously [15] while the O/UKG/34/2001 field virus has been characterized in separate studies in pigs [28,29] and cattle [27].

The cell culture adapted O1K B64 did not cause clinical signs of FMD in pigs as was observed previously in cattle [15]. Viral RNA in serum was only found at low levels within one pig on a single day. It seems that this HS-binding virus either does not reach the blood or else is rapidly cleared from the circulation and thus does not get the opportunity to spread and replicate within the inoculated animals. In contrast, both the O1K/O-UKG and the O1K/A-TUR chimeric viruses, which have capsid proteins from field strains but are otherwise identical to the $01 \mathrm{~K}$ B64 virus, caused classical symptoms of FMDV infection in pigs, albeit at different levels. The O1K/O-UKG virus caused severe disease in pigs with $100 \%$ morbidity and an overall mortality of $50 \%$, indeed for the in-contact pigs mortality was $75 \%$. These results are similar to those seen in cattle [15] except that no mortality was observed in this species. In contrast, the O1K/A-TUR chimera showed much lower disease impact in pigs (only 1 of the 4 inoculated animals showed clinical signs) whereas in cattle 5 of 6 animals in the group exposed to this chimera became infected within a similar experiment [15]. Furthermore, one of the pigs seemed to become infected with this chimeric virus late in the experiment (as judged by the appearance of viral RNA in serum, see Figure $2 b$ ) but due to the short time perspective (the experiment was terminated after 10 days) development of clinical disease and possible transmission to pen-mates could not be followed, however, signs of myocardial degeneration were observed in this pig at autopsy. Therefore a higher morbidity rate for the pigs might have been seen if this experiment had been prolonged. Pigs inoculated with the field strain $\mathrm{O}$ UKG/34/2001 demonstrated severe clinical disease and high levels of viral RNA in serum. Clinical signs (lameness, severe vesicular lesions along coronary bands and heel bulb area and to a lesser extent in the mouth region) closely resembled symptoms described in earlier studies $[28,29]$ and levels of viral RNA in serum (peak concentrations of $10^{10}$ genome copies $/ \mathrm{mL}$ ) were very high (c.f up to $10^{9.7}$ genomes $/ \mathrm{mL}$ determined by Quan et al. [28]). This virus strain causes more severe disease in pigs than in cattle $[27,29]$.

A corresponding serotype specific antibody response was developed in all pigs that became systemically infected and then survived through the acute phase, irrespective of which virus or derivative the individual pig was exposed to.

In this study we saw acute deaths in pigs exposed to the O1K/O-UKG chimera. Three out of 4 contact pigs were found dead in the pen shortly after initial clinical signs were recorded. High amounts of viral RNA was found in the heart (myocardium) of these pigs. Fatal FMD is often associated with myocarditis, a phenomenon recognized to occur only in young animals. In these cases macroscopical examination of the heart often reveals a soft, flaccid heart with white or greyish stripes, so-called "tiger heart". However, there may be no significant macroscopical lesions, but virus can usually be isolated from the myocardium [1]. It seems likely that acute myocarditis was the cause of death in pigs in our study, even though no myocardial degeneration was visually detected in these pigs (id nos. 17, $22,23,24)$, due to the high levels of FMDV RNA detected in the heart of these animals. During the viraemic phase of disease, virus can be found in all organs; therefore organs should be regarded as sites of active viral replication only when they show a higher concentration of virus or viral RNA than found in blood at the same time [1]. In the present study, the level of FMDV RNA in corresponding serum and heart tissue samples was similar (as judged by Ct levels). However, during the RNA preparation procedure (for extraction and RT-PCR) the heart tissue samples were diluted 1:10 for homogenization and therefore the viral load in heart tissue should be regarded as even higher than in serum. This indicates a specific secondary replication of virus in the heart musculature of these pigs and was only observed in pigs that died in the acute phase of infection. It is not clear why the O1K/O-UKG chimeric virus should cause a higher rate of death than the parental field strain (since the level of viral RNA reached in serum was similar) but this may just be a consequence of the relatively small number of animals involved. Interestingly, one contact pig (id no. 11) from Group 2, infected with the other chimeric virus (O1K/A-TUR), showed macroscopic lesions compatible with myocardial degeneration and could have been a candidate for myocardial infarction. Viral RNA in oral swab samples was found at low levels in several pigs which were not going through a systematic FMDV infection, especially in pigs from Group 2 inoculated with the O1K/A-TUR chimera. This probably represents background environmental virus that had been inhaled and trapped in the respiratory tract originating from the single pig which developed clinical disease.

In Groups 3 and 4, the majority of the pigs inoculated with virus (the O1K/O-UKG chimera or the O-UKG/34/ 2001 field strain, respectively) developed disease on dpi 2-4. However, 2 pigs (id nos. 20 and 29) developed disease at dpi 9 and dpi 5, respectively, i.e. in the same time interval as the in-contacts, which developed disease from dpi 5 and onwards. Whether these 2 inoculated pigs responded late to the inoculated virus or should be regarded as contact infected pigs cannot be determined.

Continuing epidemics of FMDV infection in large parts of the world, remind us of the necessity of still putting effort into FMDV research in order to broaden our knowledge regarding the pathogenicity of this virus and 
the specific host-virus interactions which underlie this. The chimeric viruses used here provide a useful tool to determine the influence of the capsid proteins alone on the properties of virus and the experiments in the natural host animals are critical for understanding how these factors determine the outcome of virus infection. Since the O1K B64, O1K/O-UKG and O1K/A-TUR viruses are genetically identical except for their capsid protein coding regions, but differ markedly in their ability to induce disease, the clear conclusion from these studies is that these capsid proteins are determinants of FMDV pathogenicity in swine.

\section{Competing interests}

The authors declare that they have no competing interests.

\section{Authors' contributions}

LL planned and performed the animal experiment and analysed the data. TJ designed and assembled the chimeric FMDV cDNA clones. AB participated in the overall planning of the experiment. GJB rescued the chimeric FMDVs, participated in the overall planning of the animal experiment and analysed the data. LL and GJB wrote the manuscript. All authors commented and approved the final manuscript.

\section{Acknowledgements}

The authors thank Jane Borch, Jani Christiansen, Inge Nielsen and Preben Normann for technical assistance. Likewise Heidi Lehman, Rikke Arnov and Ove Bille are thanked for taking excellent care of the animals and for their assistance during the experiment.

This study was financially supported in part by core funds from within DTUVET and TJ was supported by DEFRA (U.K.) project no. SE2720.

\section{Author details}

'National Veterinary Institute, Technical University of Denmark, Lindholm, Kalvehave DK-4771, Denmark. ${ }^{2}$ Institute for Animal Health, Pirbright, Woking, Surrey GU24 ONF, United Kingdom.

Received: 4 April 2012 Accepted: 27 April 2012

Published: 24 May 2012

\section{References}

1. Alexandersen S, Zhang Z, Donaldson Al, Garland AJ: The pathogenesis and diagnosis of foot-and-mouth disease. J Comp Pathol 2003, 129:1-36.

2. Orsel K, Roest HI, Elzinga-Bril EM, van Hemert-Kluitenberg F, Dekker A: Detection of foot-and-mouth disease virus in infected pigs by RT-PCR four weeks after challenge. Vet Rec 2008, 162:753-754.

3. Mohamed F, Swafford S, Petrowski H, Bracht A, Schmit B, Fabian A, Pacheco JM, Hartwig E, Berninger M, Carrillo C, Mayr G, Moran K, Kavanaugh D, Leibrecht $\mathrm{H}$, White W, Metwally S: Foot-and-mouth disease in feral swine: susceptibility and transmission. Transbound Emerg Dis 2011, 58:358-371.

4. Rodriguez-Calvo T, Diaz-San Segundo F, Sanz-Ramos M, Sevilla N: A replication analysis of foot-and-mouth disease virus in swine lymphoid tissue might indicate a putative carrier stage in pigs. Vet Res 2011, 42:22

5. Belsham GJ: Translation and replication of FMDV RNA. Curr Top Microbiol Immunol 2005, 288:43-70.

6. Jackson T, Sheppard D, Denyer M, Blakemore W, King AM: The epithelial integrin alphavbeta6 is a receptor for foot-and-mouth disease virus. J Virol 2000, 74:4949-4956.

7. Monaghan P, Gold S, Simpson J, Zhang Z, Weinreb PH, Violette SM, Alexandersen S, Jackson T: The alpha(v)beta6 integrin receptor for Footand-mouth disease virus is expressed constitutively on the epithelial cells targeted in cattle. J Gen Virol 2005, 86:2769-2780.

8. O'Donnell V, Pacheco JM, Gregg D, Baxt B: Analysis of foot-and-mouth disease virus integrin receptor expression in tissues from naive and infected cattle. J Comp Pathol 2009, 141:98-112.

9. Jackson T, Mould AP, Sheppard D, King AM: Integrin alphavbeta1 is a receptor for foot-and-mouth disease virus. J Virol 2002, 76:935-941.
10. Jackson T, Clark S, Berryman S, Burman A, Cambier S, Mu D, Nishimura S, King AM: Integrin alphavbeta8 functions as a receptor for foot-and-mouth disease virus: role of the beta-chain cytodomain in integrin-mediated infection. J Virol 2004, 78:4533-4540.

11. Johns HL, Berryman S, Monaghan P, Belsham GJ, Jackson T: A dominantnegative mutant of rab5 inhibits infection of cells by foot-and-mouth disease virus: implications for virus entry. J Virol 2009, 83:6247-6256.

12. Jackson T, Ellard FM, Ghazaleh RA, Brookes SM, Blakemore WE, Corteyn AH, Stuart DI, Newman JW, King AM: Efficient infection of cells in culture by type $\mathrm{O}$ foot-and-mouth disease virus requires binding to cell surface heparan sulfate. J Virol 1996, 70:5282-5287.

13. Fry EE, Lea SM, Jackson T, Newman JW, Ellard FM, Blakemore WE, AbuGhazaleh R, Samuel A, King AMQ, Stuart DI: The structure and function of a foot-and-mouth disease virus-oligosaccharide receptor complex. EMBO J 1999, 18:543-554.

14. Sa-Carvalho D, Rieder E, Baxt B, Rodarte R, Tanuri A, Mason PW: Tissue culture adaptation of foot-and-mouth disease virus selects viruses that bind to heparin and are attenuated in cattle. J Virol 1997, 71:5115-5123.

15. Bøtner A, Kakker NK, Barbezange C, Berryman S, Jackson T, Belsham GJ: Capsid proteins from field strains of foot-and-mouth disease virus confer a pathogenic phenotype in cattle on an attenuated, cell-culture-adapted virus. J Gen Virol 2011, 92:1141-1151.

16. Dunn CS, Donaldson Al: Natural adaption to pigs of a Taiwanese isolate of foot-and-mouth disease virus. Vet Rec 1997, 141:174-175.

17. Beard CW, Mason PW: Genetic determinants of altered virulence of Taiwanese foot-and-mouth disease virus. J Virol 2000, 74:987-991.

18. Knowles NJ, Davies PR, Henry T, O'Donnell V, Pacheco JM, Mason PW: Emergence in Asia of foot-and-mouth disease viruses with altered host range: characterization of alterations in the $3 \mathrm{~A}$ protein. J Virol 2001, 75:1551-1556

19. Oem JK, Yeh MT, McKenna TS, Hayes JR, Rieder E, Giuffre AC, Robida JM, Lee KN, Cho IS, Fang X, Joo YS, Park JH: Pathogenic characteristics of the Korean 2002 isolate of foot-and-mouth disease virus serotype $\mathrm{O}$ in pigs and cattle. J Comp Pathol 2008, 138:204-214.

20. Zibert A, Maass G, Strebel K, Falk MM, Beck E: Infectious foot-and-mouth disease virus derived from a cloned full-length cDNA. J Virol 1990, 64:2467-2473

21. Falk MM, Sobrino F, Beck E: VPg gene amplification correlates with infective particle formation in foot-and-mouth disease virus. J Virol 1992, $66: 2251-2260$

22. Ellard FM, Drew J, Blakemore WE, Stuart DI, King AM: Evidence for the role of His-142 of protein $1 \mathrm{C}$ in the acid-induced disassembly of foot-andmouth disease virus capsids. J Gen Virol 1999, 80:1911-1918.

23. Rieder E, Bunch T, Brown F, Mason PW: Genetically engineered footand-mouth disease viruses with poly $(C)$ tracts of two nucleotides are virulent in mice. J Virol 1993, 67:5139-5145.

24. Piccone ME, Rieder E, Mason PW, Grubman MJ: The foot-and-mouth disease virus leader proteinase gene is not required for viral replication. J Virol 1995, 69:5376-5382.

25. Baranowski E, Sevilla N, Verdaguer N, Ruiz-Jarabo CM, Beck E, Domingo E: Multiple virulence determinants of foot-and-mouth disease virus in cell culture. J Virol 1998, 72:6362-6372.

26. van Rensburg HG, Henry TM, Mason PW: Studies of genetically defined chimeras of a European type A virus and a South African Territories type 2 virus reveal growth determinants for foot-and-mouth disease virus. J Gen Virol 2004, 85:61-68.

27. Stenfeldt C, Heegaard PM, Stockmarr A, Tjornehoj K, Belsham GJ: Analysis of the acute phase responses of Serum Amyloid A, Haptoglobin and Type 1 Interferon in cattle experimentally infected with foot-and-mouth disease virus serotype O. Vet Res 2011, 42:66.

28. Quan M, Murphy CM, Zhang Z, Alexandersen S: Determinants of early foot-and-mouth disease virus dynamics in pigs. J Comp Pathol 2004, 131:294-307.

29. Alexandersen S, Quan M, Murphy C, Knight J, Zhang Z: Studies of quantitative parameters of virus excretion and transmission in pigs and cattle experimentally infected with foot-and-mouth disease virus. J Comp Pathol 2003, 129:268-282.

30. Have $\mathrm{P}$, Jensen MH: Detection of antibodies to foot-and-mouth disease virus type $O$ by enzyme-linked immunosorbent assay (ELISA). In Proceedings Research Group of the session of the Standing Technical Committee of the 
European Commission for the Control of Foot-and-Mouth Disease. Lelystad, Netherlands: 1983. Appendix VIII: pp. 44-51.

31. Balinda SN, Tjornehoj K, Muwanika VB, Sangula AK, Mwiine FN, Ayebazibwe C, Masembe C, Siegismund HR, Alexandersen S: Prevalence estimates of antibodies towards foot-and-mouth disease virus in small ruminants in Uganda. Transbound Emerg Dis 2009, 56:362-371.

32. Reid SM, Grierson SS, Ferris NP, Hutchings GH, Alexandersen S: Evaluation of automated RT-PCR to accelerate the laboratory diagnosis of foot-andmouth disease virus. J Virol Methods 2003, 107:129-139.

33. Jamal SM, Ferrari G, Ahmed S, Normann P, Belsham GJ: Genetic diversity of foot-and-mouth disease virus serotype $\mathrm{O}$ in Pakistan and Afghanistan, 1997-2009. Infect Genet Evol 2011, 11:1229-1238.

34. van Roermund $\mathrm{HJ}$, Eble PL, de Jong MC, Dekker A: No between-pen transmission of foot-and-mouth disease virus in vaccinated pigs. Vaccine 2010, 28:4452-4461.

doi:10.1186/1297-9716-43-46

Cite this article as: Lohse et al: Capsid coding sequences of foot-andmouth disease viruses are determinants of pathogenicity in pigs.

Veterinary Research 2012 43:46.

\section{Submit your next manuscript to BioMed Central and take full advantage of:}

- Convenient online submission

- Thorough peer review

- No space constraints or color figure charges

- Immediate publication on acceptance

- Inclusion in PubMed, CAS, Scopus and Google Scholar

- Research which is freely available for redistribution 